\title{
Isochoric ideality in jammed random packings of non-spherical granular matter
}

\author{
Andriy V. Kyrylyuk, ${ }^{*}$ Marie Anne van de Haar, Laura Rossi, Alan Wouterse and Albert P. Philipse \\ Received 30th July 2010, Accepted 9th December 2010 \\ DOI: 10.1039/c0sm00754d
}

Randomly packed particles in granular matter, ubiquitous in nature and industry, usually defy simple predictions for the optimal amorphous packing density because jammed granules are strongly correlated. While mixing different granular shapes seems to be a further complication, we discovered in simulations that binary rodsphere mixtures harbor a surprisingly simple dependence of packing volume fraction on mixture composition. This isochoric ideality covers the entire composition range and is experimentally validated by mixtures of sphero-cylindrical TicTac sweets and spherical beads: their joint random packing volume is indeed independent of the segregation state. Isochoric ideality occurs in a rod-shape range that includes the unique aspect ratio, which universally maximizes rod-sphere packing densities and suggests a novel amorphous analog of a plastic crystal, namely rod-sphere mixtures with completely uncorrelated particle orientations.

The puzzle of dense amorphous particle packings has caught the close attention of mathematicians and scientists from many different disciplines. In particular, non-spherical particles and their mixtures, jammed into random packings, are abundant and occur on widely different length scales. Macroscopic instances of anisotropic granular matter, among many others, are the grains in solidifying igneous rocks, various grains in food (rice, pasta's, TicTac sweets, etc.), ceramic bulk powders, catalyst pellets, and reinforcing fibers in industry. ${ }^{1-6}$ On the sub-micron length scale anisometric colloids such as rods and platelets form dense random packings and glasses, ${ }^{4,-13}$ while protein filaments may randomly pack in animal cells. ${ }^{14}$

Essential questions are how tight one can pack particles in jammed amorphous packings and how to optimize the corresponding random close packing (RCP) density. In quest of the optimal or densest random packing, particle non-sphericity has proven to be an effective means to maximize the RCP density. Recent studies on random packing of sphero-cylinders ${ }^{15}$ and ellipsoids ${ }^{16,17}$ revealed an intriguing non-monotonic dependence of the RCP density on the particle elongation. Starting from the Bernal random sphere packing, the RCP density first raises to a maximum for nearly spherical particles and only beyond this maximum the random packing density monotonically decreases with particle aspect ratio. So far, this

Van't Hoff Laboratory for Physical and Colloid Chemistry, Debye Institute for Nanomaterials Science, Utrecht University, Padualaan 8, 3584, CH, Utrecht, The Netherlands. E-mail: a.kyrylyuk@gmail.com peculiar maximum has only been observed for monodisperse granules ${ }^{15-17}$ and colloids. ${ }^{9}$ As polydispersity in size and shape is often unavoidable, one important question is how to optimize packing densities of granular mixtures, which ideally could be predicted from densities of the monodisperse components. It is therefore of fundamental and practical interest to investigate for such mixtures the existence of a density maximum and the universality, if any, in its location or magnitude.

We uncover the universality in jamming of rather short spherocylinders of length $L$ (including two hemi-spherical caps at both ends) and diameter $D$ by investigating the RCP of their binary mixtures with spheres of the same diameter $D$. A binary rod-sphere mixture is an ideal model system to verify any universality. Though it is a relatively simple system that consists of only two components, it gives extra control parameters to explore the jamming "phase" diagram. On the other hand, the binary mixtures in our study are sufficiently complex to reveal characteristics of polydisperse packings of nonspheres in general. ${ }^{18}$

In addition to the universality, we report on the ideality in random jammed packings of hard non-spherical particles. The concept of ideality of a system of particles is one of the most dominating ideas in science. The paradigm of an ideal gas where the particles are completely uncorrelated, which is valid for very dilute systems, led to tremendous advances in statistical physics. The manifestation of such an ideal random dense packing, which could serve as a reference system for real random packings governed by statistical geometry, could result in much better understanding of the nature of the jamming transition and the mechanism of the formation of the random jammed packings. Unfortunately, no ideality in jamming of either monodisperse or polydisperse hard spheres is revealed due to many-body interactions. ${ }^{3,19-22}$ However, a packing of pure spheres is rather the exception than the rule - a real system usually consists of non-spherical particles. It is therefore the random jammed packing of non-spherical particles, which is a much broader class of packings, where one has to seek ideality in any sense. The potential ideality is then purely a result of the non-sphericity of the particles that posses the additional rotational degrees of freedom.

We simulate random packing of binary rod-sphere mixtures (Fig. 1), which are computationally relatively easy to handle and which have experimental instances (Fig. 4). One would expect that non-sphericity and polydispersity always complicate a random packing problem due to the additional rotational degrees of freedom and competing length scales. However, we discovered that mixtures 


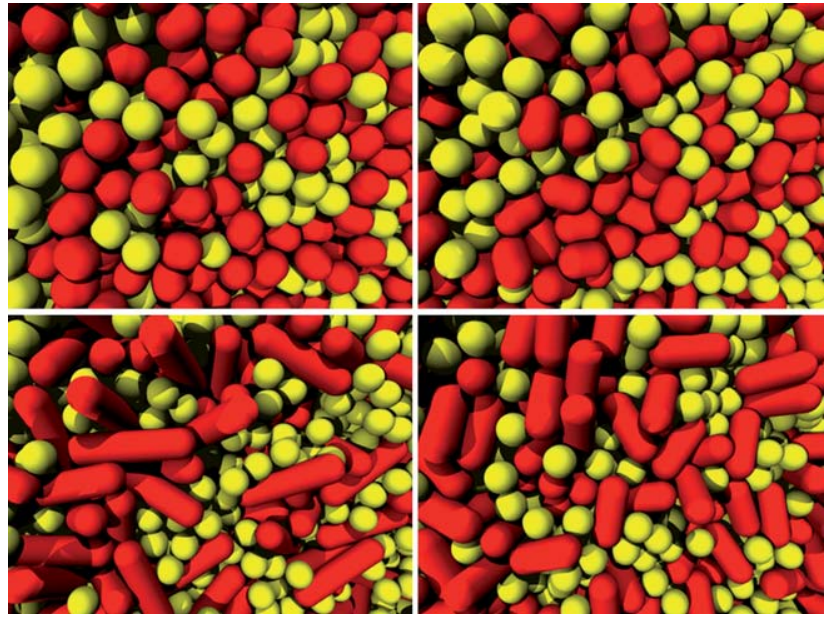

Fig. 1 Computer-generated random jammed packings of binary mixtures of $40 \%$ spheres and $60 \%$ sphero-cylinders by volume for several sphero-cylinder aspect ratios $L / D$, where $L$ is the length of the spherocylinders (including two hemi-spherical caps at both ends) and $D$ is their diameter. Clockwise from top left: $L / D=1.2, L / D=1.5, L / D=2.5$, and $L / D=4$

of spheres and sphero-cylinders exhibit an unexpected simplicity, namely a decoupling of particle positions and orientations. The position-orientation decoupling entails an ideal isochoric mixing law for the magnitude of the uncovered packing density maxima and universality in their location, as explained in what follows.

The packings are generated via the mechanical contraction method $(\mathrm{MCM}),{ }^{15,17}$ i.e. the step-wise isotropic contraction of an initially dilute random arrangement of non-overlapping a-thermal particles in a cubic simulation box with periodic boundary conditions. Particle overlaps are removed by an efficient sequence of particle orientations and translations. ${ }^{15,17}$ The MCM yields jammed packings that reproduce the experimental random packing densities of rods and spheres..$^{15}$ Here, we apply the MCM to a binary mixture of in total 5000 particles, comprising frictionless sphero-cylinders and spheres of the same diameter $D$. The resulting jammed packings are amorphous, without any crystalline or liquid crystalline order (Fig. 1). The absence of any long-range order is clearly seen from the visual inspection of the obtained packing structures, which can also be indicated by the radial distribution function characteristic for amorphous structures and an essentially zero value of the nematic order parameters for all studied jammed packings. ${ }^{17,23} \mathrm{~A}$ striking feature of the mixture packing densities in Fig. 2, where each data point is a result of averaging over five to ten different simulation runs, is that they are non-monotonic functions of rod aspect ratio for all mixture compositions expressed in terms of the relative rod volume fraction $x=\phi_{\mathrm{r}} / \phi=N_{\mathrm{r}} V_{\mathrm{r}} /\left(N_{\mathrm{r}} V_{\mathrm{r}}+N_{\mathrm{s}} V_{\mathrm{s}}\right)$. Here, $\phi_{i}$ is the volume fraction of one of the two components $i \equiv \mathrm{r}$ (rods) and $i \equiv \mathrm{s}$ (spheres), $\phi=\phi_{\mathrm{r}}+\phi_{\mathrm{s}}$ is the total packing fraction, $N_{i}$ is the number of particles of type $i$, and $V_{i}$ is the volume of one particle of type $i$. All rod-sphere mixture compositions exhibit a pronounced density maximum close to the Bernal sphere packing. So, clearly this maximum is not a unique feature of monodisperse packings but a universal property of mixtures (in any case of sphero-cylinders), with the maximum always located at the particle aspect ratio $L / D=1.5$. Apart from this aspect ratio, another special aspect ratio is $L / D \approx 4$ where within the

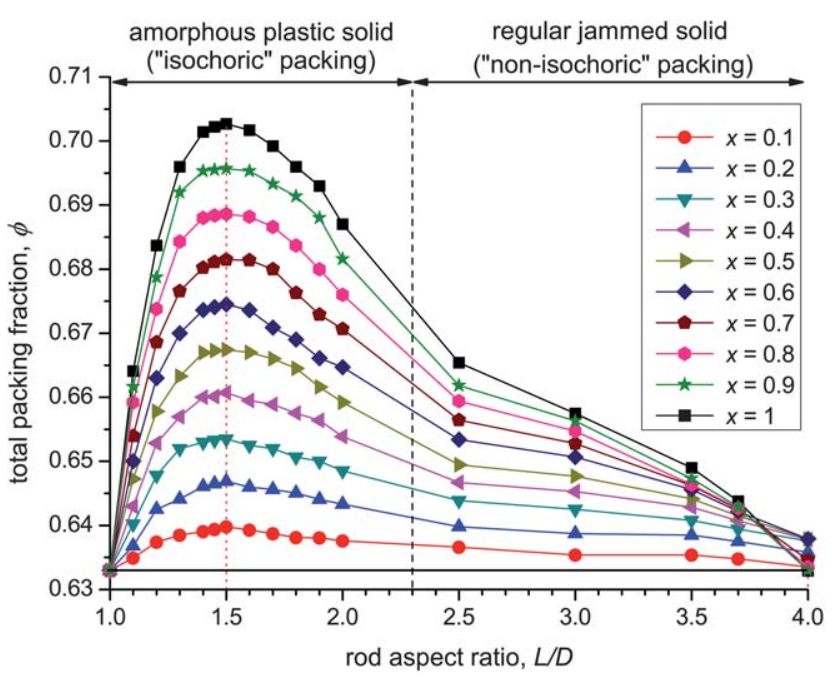

Fig. 2 The MCM computer simulation jamming diagram of binary mixtures of hard frictionless spheres and sphero-cylinders in the entire range of pure sphere to pure sphero-cylinder packing as a function of the rod shape anisotropy $L / D$ for various mixture compositions expressed in terms of the relative rod volume fraction $x=\phi_{\mathrm{r}} / \phi$, where $\phi=\phi_{\mathrm{r}}+\phi_{\mathrm{s}}$ is the total packing volume fraction and $\phi_{i}$ is the volume fraction of species $i$ with $i \equiv \mathrm{r}$ for the rods (sphero-cylinders) and $i \equiv \mathrm{s}$ for the spheres. The horizontal black line corresponds to the Bernal random sphere packing density $\phi=0.633$.

simulation uncertainty the mixture packing densities all intersect at the density of the Bernal sphere packing $\phi=0.633$.

The density maxima in Fig. 2 are not caused by long-range order in the packings but manifest competition between local caging and excluded volume effects. Jammed particles are arrested by a cage of contacting neighbors; a short sphero-cylinder can be oriented to fit better in this cage, which raises the density. Beyond the density maximum the growth of the orientationally averaged excluded volume with increasing aspect ratio is the dominating effect that

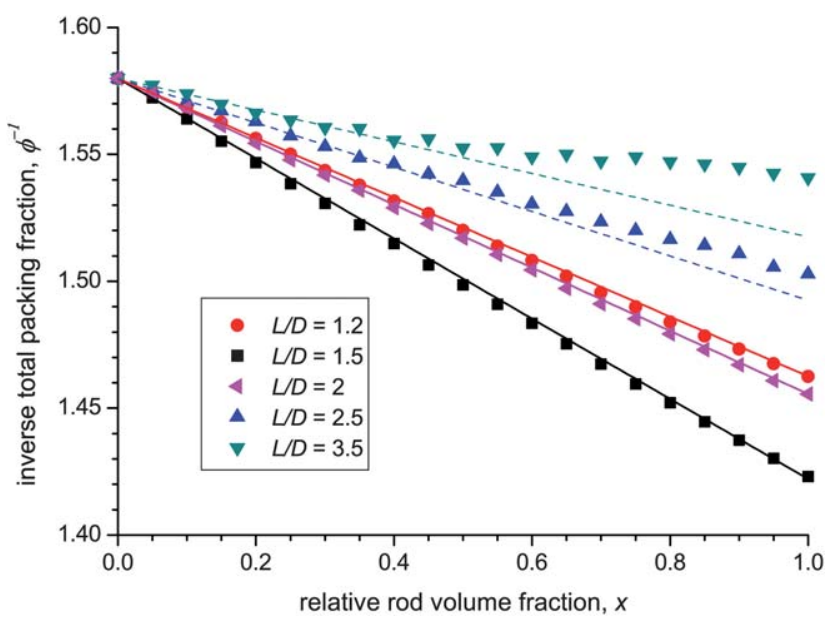

Fig. 3 The inverse of the total RCP fraction $\phi$ of a rod-sphere mixture as a function of the relative rod volume fraction for several rod aspect ratios. The solid and broken lines represent the packing densities expected from the ideal rule of mixtures for $\phi^{-1}$. 
drives the packing density down (Fig. 2). At the aspect ratio $L / D \approx 4$ the competing local caging and excluded volume effects apparently balance each other since within the simulation uncertainty all the packing curves intersect at one point so that the RCP of rods of this specific elongation approximately equals to the Bernal random sphere packing.

Remarkably, in the shape range near the density maximum $(1 \leq L /$ $D \lesssim 2.3$ ), the inverse total density $\phi^{-1}$ of binary mixtures depends linearly on the mixture composition $x$. The linearity of the corresponding packing curves (see Fig. 3) means per se that jammed packings of short rod-sphere particulate systems obey the simple law of mixtures for the inverse packing fraction $1 / \phi=x / \phi_{\mathrm{r}}^{\mathrm{RCP}}+(1-x) /$ $\phi_{\mathrm{s}}^{\mathrm{RCP}}$, where $\phi_{\mathrm{r}}^{\mathrm{RCP}}$ and $\phi_{\mathrm{s}}^{\mathrm{RCP}}$ are the RCP densities of the jammed systems of pure rods and spheres, respectively. When inverse total packing densities change linearly going from pure spheres to pure rods, the segregation state of the binary mixture is irrelevant. Stated differently, the mathematical form of the rule of mixtures for $\phi^{-1}$ implies that the total volume remains constant when two separate volumes of randomly packed rods and spheres are shaken to a homogeneous mixture. We refer to this constancy as isochoric ideality of granular mixtures. For such isochoric granular materials the packing volume is additive - the volume of the box $V_{\mathrm{r}+\mathrm{s}}{ }^{\text {box }}$ occupied by the mixed binary rod-sphere system at RCP equals to the total volume filled by its two jammed components that are packed independently in two different boxes with volumes $V_{\mathrm{r}}^{\text {box }}$ and $V_{\mathrm{s}}^{\text {box }}$ or that are segregated, e.g. in layers in one box, $V_{\mathrm{r}+\mathrm{s}}{ }^{\text {box }}=V_{\mathrm{r}}^{\mathrm{box}}+V_{\mathrm{s}}^{\mathrm{box}}$. The independence of the total packing volume of the segregation state or dispersion/positions of rods in the rod-sphere packing means that the two subsystems of the rods and spheres are in some sense decoupled. Jammed packings of binary or polydisperse spheremixtures $^{19-22}$ are strongly non-ideal in this respect: total packing densities are non-monotonic functions of mixture composition. For ideality to occur some correlation must vanish and for a binary rodsphere mixture this most likely is the correlation between the rod positions and orientations because of the 'isochoric' behavior of the mixture. For a few rods in a sea of randomly packed spheres decoupling of positions and orientations is obvious. However, the striking and somewhat counter-intuitive inference from Fig. 3 is the persistence of this decoupling over the entire composition range. Note the interesting analogy with a plastic crystal, ${ }^{24}$ which has a similar
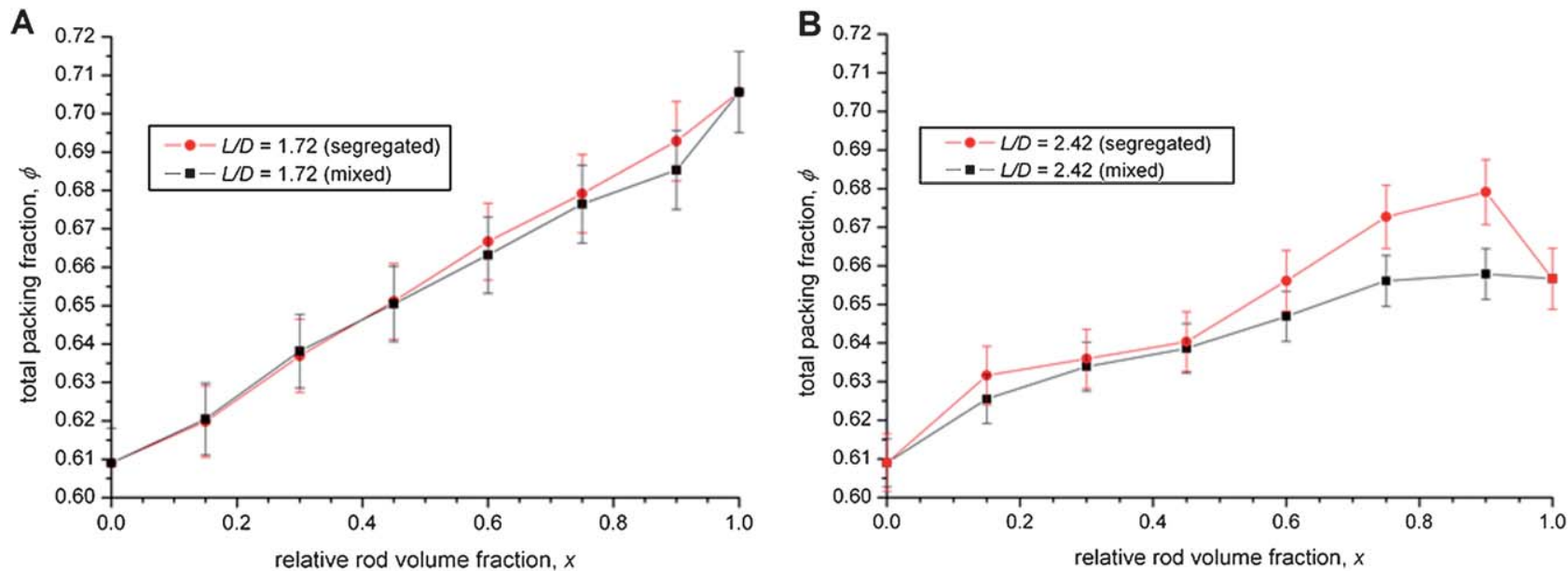

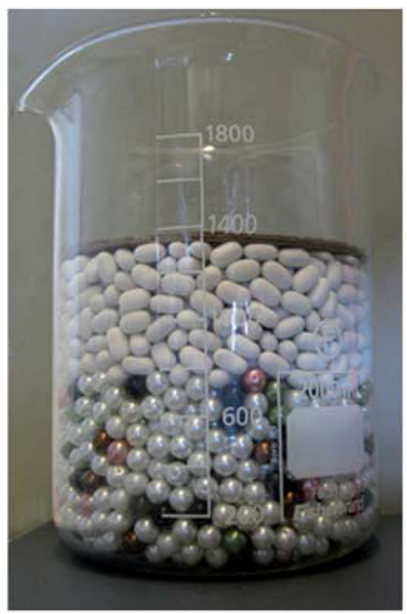

segregated

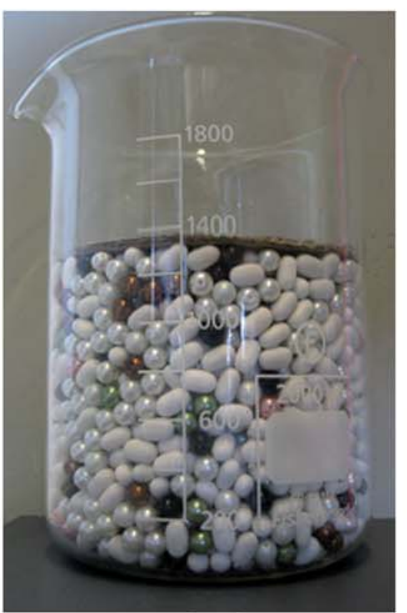

mixed

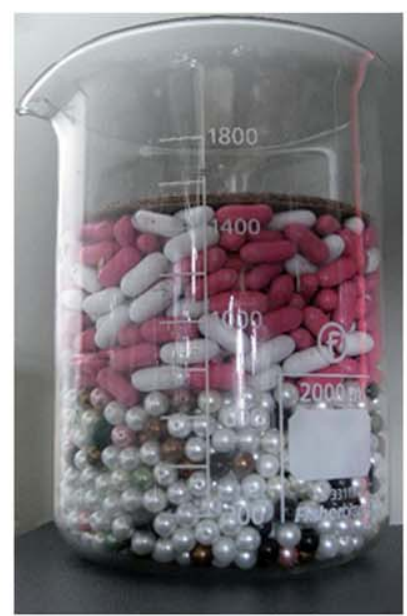

segregated

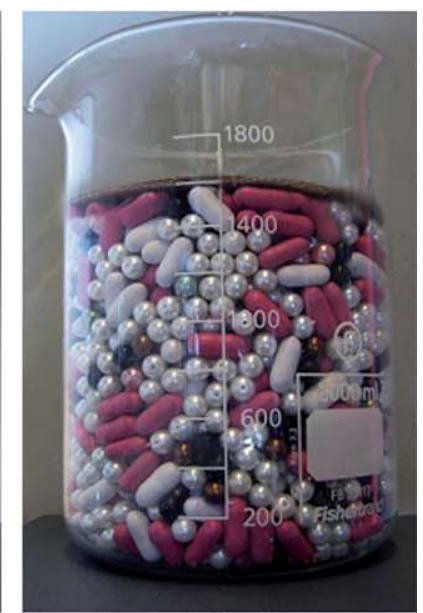

mixed

Fig. 4 Gentle shaking and tapping of spherical beads and sphero-cylindrical sweets in graded beakers yields fairly reproducible random packing densities $\phi$, plotted versus relative rod volume fraction $x$. TicTacs (A) with aspect ratio $L / D=1.72$ clearly exhibit isochoric ideality: both segregated and mixed packings have almost the same linear dependence of $\phi$ on $x$. The aspect ratio $L / D=2.42$ for licorices (B) already induces clear deviations from isochoric mixing. Photographs are shown for a mixture composition $x=0.6$. Note the equality of the total packing volumes of the segregated and mixed state in A, whereas B displays an excess mixing volume. 
position-orientation decoupling, be it for positions that form an FCC lattice. In our rod-sphere mixtures long-range positional order is absent so that we can suggest isochoric mixtures as the amorphous counterpart of a plastic crystal.

Isochoric ideality also closely ties up with the universality in the location of the density maxima in Fig. 3. The linearity of the packing curves as a function of mixture composition shown in Fig. 3 requires the position of all the density maxima in Fig. 2 to hold for one unique rod aspect ratio. Otherwise, the packing curves would exhibit a nonmonotonic dependence on the relative volume fraction of rods in a mixture. Both ideality and universality are caused by the decoupling of particle positions and orientations. Indeed, due to such decoupling, the optimal orientation of a rod in the cage of neighboring particles that leads to the density maximum occurs for the same rod aspect ratio, irrespectively of the relative amount of other rods and their positions.

We verified the existence of isochoric ideality for granular matter also experimentally by measuring random packing densities for spherical beads and sphero-cylindrical sweets, both in segregated and mixed state (Fig. 4). The aspect ratio $L / D=1.72$ of TicTacs is close to the maximum density value $L / D=1.5$ in Fig. 2 and, indeed, we find binary packing densities significantly above that for spheres (Fig. 4A). The higher aspect ratio $L / D=2.42$ of sphero-cylindrical licorices (Fig.4B) reduces the total packing fraction in comparison to the TicTacs, completely in line with the simulation results in Fig. 2 and 3. Jammed TicTac-sphere mixtures clearly exhibit isochoric ideality: both segregated and mixed packings (Fig. 4A) have almost the same linear dependence of the total packing fraction on mixture composition. The total packing volume occupied by the mixed rod-like TicTacs and spheres is a linear superposition of packing volumes of the two segregated components, i.e. jammed packings of the pure spheres and pure rods (Fig. 4A). From Fig. 3 we predict that the aspect ratio $L / D=2.42$ should already induce clear deviations from isochoric mixing. This prediction is distinctly confirmed by the experimental data in Fig. 4B.

The isochoric ideality in the mixing behavior of near-spheres gives a direct test to the statistical theories of anisotropic jammed matter in the spirit of the Edwards theory of dense random granular packings ${ }^{25}$ and to the free volume theories of entropy driven demixing as both spheres and sphero-cylinders represent an important class of hard convex bodies. ${ }^{26}$ The revealed amorphous plastic solids and associated with them isochoric granular materials have no mixing volume and no driving force for entropy driven particle segregation, which should be also predicted in a theory.

Provided that the dominant mixing mechanism is the geometrical void filling mechanism, ${ }^{27}$ shaking of an ideal jammed packing would leave the particle dispersion unaffected, eliminating both the conventional and reverse "Brazil-nut effect". Such ideal, isochoric or "Brazil-nut effect"-free materials would be of great technological interest since they occupy the constant volume irrespectively of particle dispersion, which in turn does not change upon sample shaking.

In conclusion, we have shown that granular rod-sphere mixtures harbor an ideal mixing law that holds over the entire composition range. This isochoric ideality, when a jammed particulate system occupies the same volume independently of the particle dispersion in the mixture, occurs in a rod-shape range that includes the packing density maxima. The position of the density maxima is universal in the sense that the optimal random packing appears for one unique rod aspect ratio regardless of the mixture composition. The revealed isochoric ideality is the manifestation of a novel amorphous analog of a plastic crystal - the jammed state where the orientational correlations vanish due to the decoupling of particle positions and orientations. The ideality and universality in the optimal random packing are purely results of particle non-sphericity and a subtle interplay between size and shape effects, which cannot be observed in binary or polydisperse sphere mixtures. Our results are relevant for practical granular mixtures such as ceramic powders or catalyst pellets: we now have a prediction for the rod aspect ratio that maximizes the solid volume fraction of a rod-sphere mixture. Moreover, the isochoric ideality may be exploited in the design of isochoric amorphous solids and jammed disordered matter with a controlled system volume unaffected by the state of mixing of the various components.

\section{References}

1 J. F. Rudge, M. B. Holness and G. C. Smith, Contrib. Mineral. Petrol., 2008, 156, 413.

2 J. V. Milewski, in Handbook of Fillers and Reinforcements for Plastics, ed. H. S. Katz and J. V. Milewski, Van Nostrand Reinhold, New York, 1978

3 M. van Hecke, J. Phys.: Condens. Matter, 2010, 22, 033101.

4 A. P. Philipse, Langmuir, 1996, 12, 1127; A. P. Philipse, Langmuir, 1996, 12, 5971, correction.

5 D. J. Cumberland and R. J. Crawford, The Packing of Particles, Elsevier, Amsterdam, 1987.

6 T. Aste and D. Weaire, The Persuit of Perfect Packing, IOP, London, 2000.

7 A. V. Kyrylyuk and P. van der Schoot, Proc. Natl. Acad. Sci. U. S. A., 2008, 105, 8221; A. V. Kyrylyuk and P. van der Schoot, Proc. Natl. Acad. Sci. U. S. A., 2008, 105, 11451, correction.

8 F. Sciortino and P. Tartaglia, Adv. Phys., 2005, 54, 471.

9 S. Sacanna, L. Rossi, A. Wouterse and A. P. Philipse, J. Phys.: Condens. Matter, 2007, 19, 376108.

10 M. C. Hermant, M. Verhulst, A. V. Kyrylyuk, B. Klumperman and C. E. Koning, Compos. Sci. Technol., 2009, 69, 656.

11 M. J. Solomon and P. T. Spicer, Soft Matter, 2010, 6, 1391.

12 N. Grossiord, et al., Polymer, 2008, 49, 2866.

13 G. Yatsenko and K. S. Schweizer, Phys. Rev. E: Stat., Nonlinear, Soft Matter Phys., 2007, 76, 041506.

14 L. Miao, et al., Proc. Natl. Acad. Sci. U. S. A., 2008, 105, 5390.

15 S. R. Williams and A. P. Philipse, Phys. Rev. E: Stat., Nonlinear, Soft Matter Phys., 2003, 67, 051301.

16 A. Donev, et al., Science, 2004, 303, 990.

17 A. Wouterse, S. R. Williams and A. P. Philipse, J. Phys.: Condens. Matter, 2007, 19, 406215.

18 H. Löwen, J. Phys.: Condens. Matter, 2001, 13, R415.

19 N. Xu and S. C. Ching, Soft Matter, 2010, 6, 2944.

20 R. S. Farr and R. D. Groot, J. Chem. Phys., 2009, 131, 244104.

21 E. K. H. Li and P. D. Funkenbusch, Mater. Sci. Eng., A, 1992, 157, 217.

22 D. He, N. N. Ekere and L. Cai, Phys. Rev. E: Stat. Phys., Plasmas, Fluids, Relat. Interdiscip. Top., 1999, 60, 7098.

23 A. Wouterse, S. Luding and A. P. Philipse, Granular Matter, 2009, 11, 169.

24 P. Bolhuis and D. Frenkel, J. Chem. Phys., 1997, 106, 666.

25 S. F. Edwards and R. B. S. Oakeshott, Physica A (Amsterdam), 1989, 157, 1080.

26 K. W. Wojciechowski, Physica A (Amsterdam), 1996, 232, 723.

27 A. Kudrolli, Rep. Prog. Phys., 2004, 67, 209. 